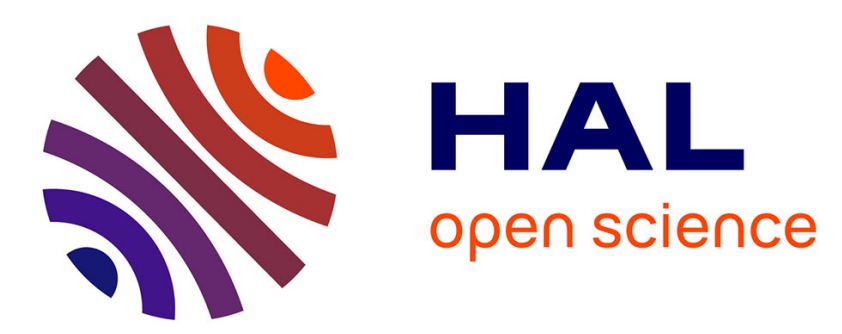

\title{
A correlation of the convective heat transfer coefficient between an air flow and a phase change material plate
}

A. de Gracia, Damien David, Albert Castell, Luisa F. Cabeza, Joseph Virgone

\section{To cite this version:}

A. de Gracia, Damien David, Albert Castell, Luisa F. Cabeza, Joseph Virgone. A correlation of the convective heat transfer coefficient between an air flow and a phase change material plate. Applied Thermal Engineering, 2013, 51 (1), pp.1245-1254. 10.1016/j.applthermaleng.2012.11.045 . hal01003072

\section{HAL Id: hal-01003072 \\ https://hal.science/hal-01003072}

Submitted on 9 Jun 2014

HAL is a multi-disciplinary open access archive for the deposit and dissemination of scientific research documents, whether they are published or not. The documents may come from teaching and research institutions in France or abroad, or from public or private research centers.
L'archive ouverte pluridisciplinaire HAL, est destinée au dépôt et à la diffusion de documents scientifiques de niveau recherche, publiés ou non, émanant des établissements d'enseignement et de recherche français ou étrangers, des laboratoires publics ou privés. 


\title{
A new correlation of the convective heat transfer coefficient between an air flow and a phase change material plate
}

\author{
Alvaro de Gracia ${ }^{1,2}$, Damien David ${ }^{2}$, Albert Castell ${ }^{1}$, Luisa F. Cabeza ${ }^{1}$, Joseph \\ Virgone $^{2}$ \\ ${ }^{1}$ GREA Innovació Concurrent, Universitat de Lleida, Edifici CREA, Pere de Cabrera s/n, 25001, \\ Lleida, Spain. Tel: +34.973.00.35.77. Email: Icabeza@diei.udl.cat \\ ${ }^{2}$ CETHIL, UMR 5008, INSA-Lyon, Université Lyon1, Bât. Sadi Carnot, 9 rue de la Physique, \\ 69621 Villeurbanne, France
}

\begin{abstract}
This paper provides a new correlation to determine the heat transfer coefficient between an air flow and a plate made of phase change material (PCM). This correlation was built for the simulation of heat storage units containing PCM plates subjected to an inlet temperature step. The presented correlation has the following form: $N u_{x, t}^{P C M}=N u^{P S M} \cdot f^{P C M}$. The first term $N u^{P S M}$ is for a plate made of traditional material. The term $f^{P C M}$ is a perturbation due to the phase change in the plate. Each term depends on 5 non dimensional parameters. One of them represents the advance in the total heating or cooling process, in order to take into account the transient evolution of the convective coefficient. The correlations are built using the Least Squares Method, from series of CDF simulation data. The shape of the perturbation $f^{P C M}$ reveals a complex evolution of the temperature repartition in the PCM plate. Finally, a nodal model of the plate has been developed in order to test the provided new correlation and other correlations available in the literature. The results obtained with the present correlation show better agreements with the CFD results, which make this correlation suitable for the simulation of PCM heat storage systems.
\end{abstract}

Key words: Convective heat transfer coefficient, correlation, phase change material, heat storage system, transient forced convection, numerical simulation.

\section{Introduction}

An efficient utilization of renewable energies in buildings could not be possible without appropriate energy storage systems. Indeed, the availability of these energies does not always coincide in time with the demand. Hence energy storage systems present a growing interest among research communities.

One possible way to store a high amount of energy in a small volume is the use of phase change materials (PCM). The latent heat which is needed for the material melting/solidification enables to store a great amount of energy within a narrow temperature range. The use of PCM in the building sector has been widely studied [1-3], both experimentally [4] and numerically [5]. The PCM can 
be integrated in composite walls [6], in heat exchangers which are included in the building ventilation system [7-9], or in ventilated facades [10,11].

This study is focused on heat exchangers made of PCM plates. The air flowing between the parallel plates exchanges heat with the PCM. The performances of such systems are evaluated with building simulations. Those simulations require simple and accurate models of the heat exchangers, such as nodal models, in which the heat transfer between the air flow and the plate is modeled by the convective coefficient.

In the literature, the existing heat transfer correlations for the flow between parallel plates are for two cases: "constant and equal wall temperatures", and "constant and equal wall heat fluxes". According to Liu et al. [12], the case of "constant and equal wall temperature" is more appropriate to model the convective heat transfer occurring between a forced flow and a PCM solid, since the wall temperatures are almost constant during the phase change. However, thermal gradients can occur in the PCM wall, especially in heat exchangers with long PCM slabs, which makes the assumption of "constant and equal wall temperature" not valid.

Therefore, no correlation has been built for the specific case of an air flow over PCM plates, so there was no optimal choice of the convective coefficient values in PCM heat exchangers. Wei et al. [13] obtained the Nusselt number from the experimental value in a plate heat exchanger (PHE). Moreover, Dolado et al. [14] used the correlation from Gnielinski [15] for internal forced convective coefficient while Hed and Bellander [16] used the Reynolds-Colburn analogy [17]. However their simulation results might be affected by erroneous convective heat transfer coefficient values. Indeed, David et al. $[18,19]$ simulated the thermal behavior of a composite PCM wall using different correlations to model the natural convection heat transfer over the wall. They spotted discrepancies in the simulation results.

In this study, a new correlation is built to determine the heat transfer coefficient between an air flow and a plate made of PCM. The correlation has two terms: one of them is for a plate without phase change, the other one is a perturbation on the Nusselt number due to the phase change. The methodology for the production of the two terms of the correlation is explained in section 2. Details about the geometry and the thermal excitation of the system are also given in this section. The two terms of the correlation are built in section 3 and 4 . The new correlation is finally implemented in a nodal model of PCM plates in section 5. Nodal simulation results with new provided correlation and with other correlations available in the literature are compared to CFD results.

\section{Methodology}

\subsection{Shape of the correlation}

The correlation which is built in this paper has the form of Eq.1, where $N u_{x, t}^{P C M}=h \cdot x / k$ is the local Nusselt number at the ordinate $x$ : 
The first term $\mathrm{Nu}^{P S M}$ is calculated for the heating/cooling process of a plate made of a traditional material which has the same thermo-physical properties of the PCM in the solid state. Since no phase change occurs in this plate, it is designated as the Phase Stabilized Material (PSM) plate.

The second term $f^{P C M}$ describes the perturbation on the Nusselt number due to the phase change in the wall. Thus, the formalism which is chosen for the expression of $\mathrm{Nu}_{x, t}^{P C M}$ enables to isolate phase change effects on the convective heat transfer through the term $f^{P C M}$.

For each term of the correlation, a list of 5 specific non-dimension parameters has been defined. The dependency of $N u^{P S M}$ and $f^{P C M}$ on those parameters are determined from series of computational fluid dynamics (CFD) simulations. The term $\mathrm{Nu}^{P S M}$ was obtained first from simulation results without phase change in the plate. The term $f^{P C M}$ was calculated from simulations with phase change in the plate.

\subsection{Geometry of the system}

The system which concerns this study is a pile of PCM plates separated by air channels (Figure 1a). The air flow between the plates is ensured by a homogeneous velocity inlet at the entrance of the channel. The heat storage/release starts with an inlet temperature step.

The geometry of the system has been simplified according to some hypotheses, in order to reduce the computational cost of the CDF simulations. First, it is assumed that the plates are sufficiently wide to get a 2 dimensional thermal behavior of the system. Then, since the geometry is periodic, only one channel surrounded by two half plates can be considered. The system represented in Figure. $1 \mathrm{~b}$ is obtained after performing these simplifications.

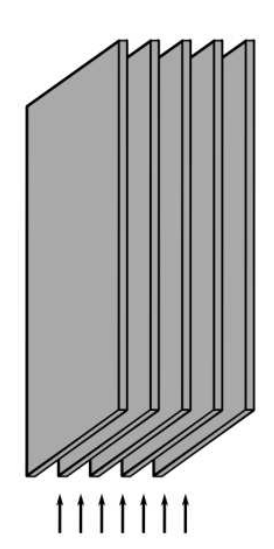

(a)

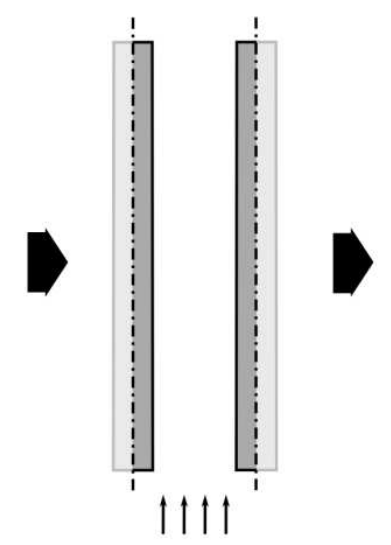

(b)

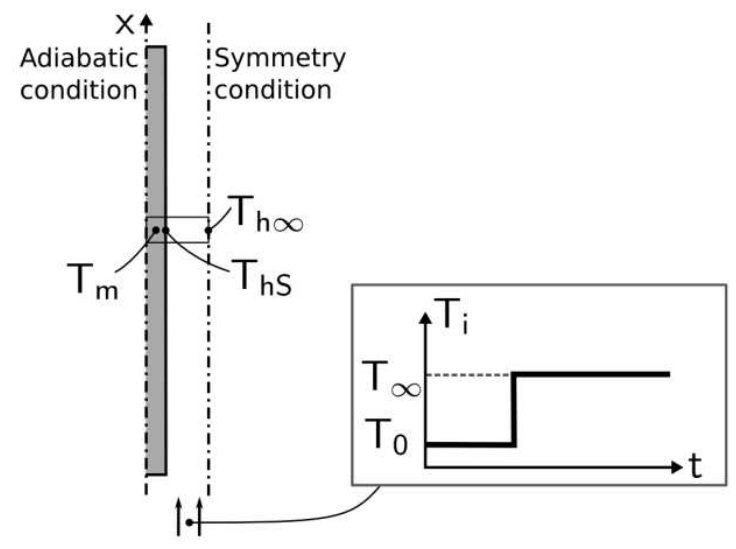

(c)

Figure 1: Simplification of the heat exchanger geometry for the model: a) actual geometry of the heat exchanger, b) Simplified geometry using the hypothesis of a periodic system and a 2D flow, c) Geometry of the model, using the hypothesis of independent boundary layers. 
Moreover, it is assumed that the thermal and velocity boundary layers are thinner than the half-width of the air channel. Since there is no mixing between the boundary layers on the two sides of the channel, only a half-channel can be considered. Even if this type of geometry does not optimize the heat transfer between the plates and the channel, it can be justified in a technical point of view because it reduces the pressure drop along the storage system. The final geometry of the model is represented in Figure 1c. For the simulations, the plate was $4 \mathrm{~m}$ high and the half-channel was $7.5 \mathrm{~cm}$ wide.

At the beginning of the simulations, the temperature is homogeneous at $T_{0}$ in all the system, and there is a steady flow in the fluid volume due to the homogeneous inlet velocity $u_{\infty}$. At $t=0$, the inlet temperature becomes equal to $T_{\infty}$. Simulations are run until the plate temperature reaches the thermal equilibrium at $T_{\infty}$. During the simulations, the half plate mid-temperature $T_{m}$, the mid-channel temperature $T_{h \infty}$, the plate surface temperature $T_{h S}$ and heat flux density $\varphi_{h s}$ are recorded at different heights and times in order to compute the heat transfer coefficients $h=\varphi_{h s} /\left(T_{h s}-T_{h \infty}\right)$ and the non-dimension parameters used in the correlations.

\subsection{Numerical Model}

The simulations have been performed with the commercial software Star$\mathrm{CCM}+$. First, a comparative study between the different fluid models proposed by the software has been carried out. Those models have been tested for a stationary forced turbulent flow along an isothermal surface. The simulation results have then been compared to the empirical correlations from Bejan [20], which are valid for Prandtl number between 0.5 and 5 :

$$
\frac{h}{\rho \cdot C p \cdot u_{\infty}}=\frac{\frac{1}{2} C_{f, x}}{0.9+\left(\frac{1}{2} C_{f, x}\right)^{0.5} \cdot(13.2 \cdot \operatorname{Pr}-10.25)}
$$

Where the drag coefficient $C_{f, x}$ is given either by:

$$
\frac{1}{2} C_{f, x}=0.0296\left(\frac{u_{\infty} \cdot x}{v}\right)^{-1 / 5}
$$

Or by:

$$
C_{f, x}=0.37\left[\log \left(\frac{u_{\infty} \cdot x}{v}\right)\right]^{-2.584}
$$

The previous correlations are valid for Reynolds number varying between $10^{5}$ to $10^{7}$ (Eq.3) and from $10^{5}$ to $10^{9}$ (Eq.4).

The best agreement with the correlations was obtained with the Segregated implicit AMG SIMPLE solver and the "realizable" $k-\varepsilon$ turbulence model. 
Hereafter, a mesh independent study was performed in order to reduce the duration of the transient simulations needed for this study. The resulting mesh consisted in 4400 rectangular cells, with a mesh refinement at the vicinity of the plate in order to capture the temperature and velocity gradients in the boundary layer.

A comparison between the final flow model and the correlations from Bejan [20] is shown in Figure 2 for different values of the inlet velocity. The results of this comparison show only a slight deviation at the starting area of the plate. This deviation is due to the presence of the laminar zone. In this zone, neither the correlation from Bejan, nor for the $k-\varepsilon$ turbulence model is adapted.

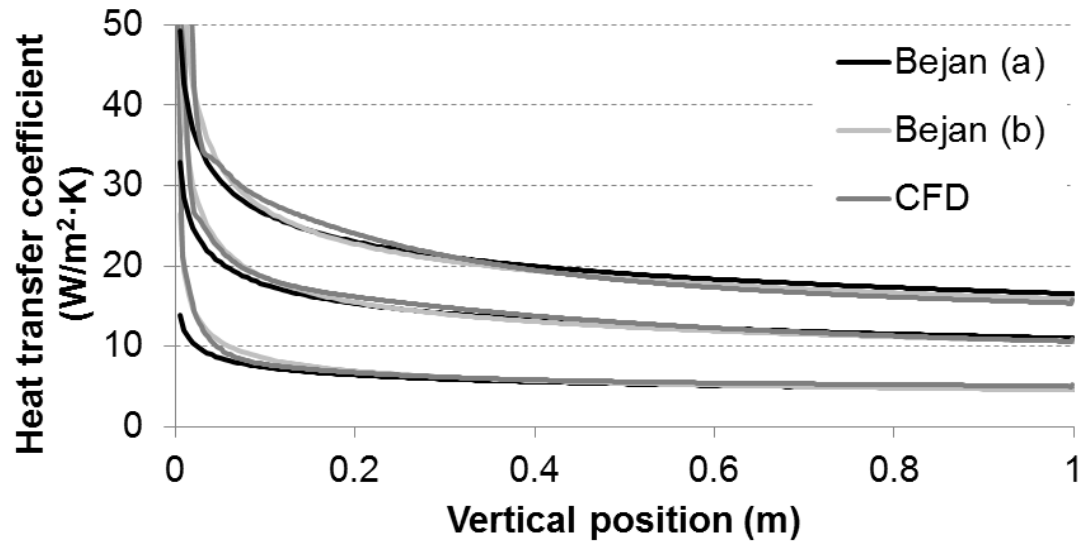

Figure 2: Fluid model validation using empirical correlation available in literature [15]

The solid model is a finite volume model with 1334 rectangular cells. The specific heat of the solid and liquid phases is equal to $C p$. The phase change was taken into account through an equivalent heat capacity [21]. During the melting or solidification, the temperature dependence of the PCM specific heat has a triangle shape centered on $T_{P C M}$ (Figure 3). This methodology was studied by Farid et al. [22] and was found to be successful in describing the heat transfer in phase change materials.

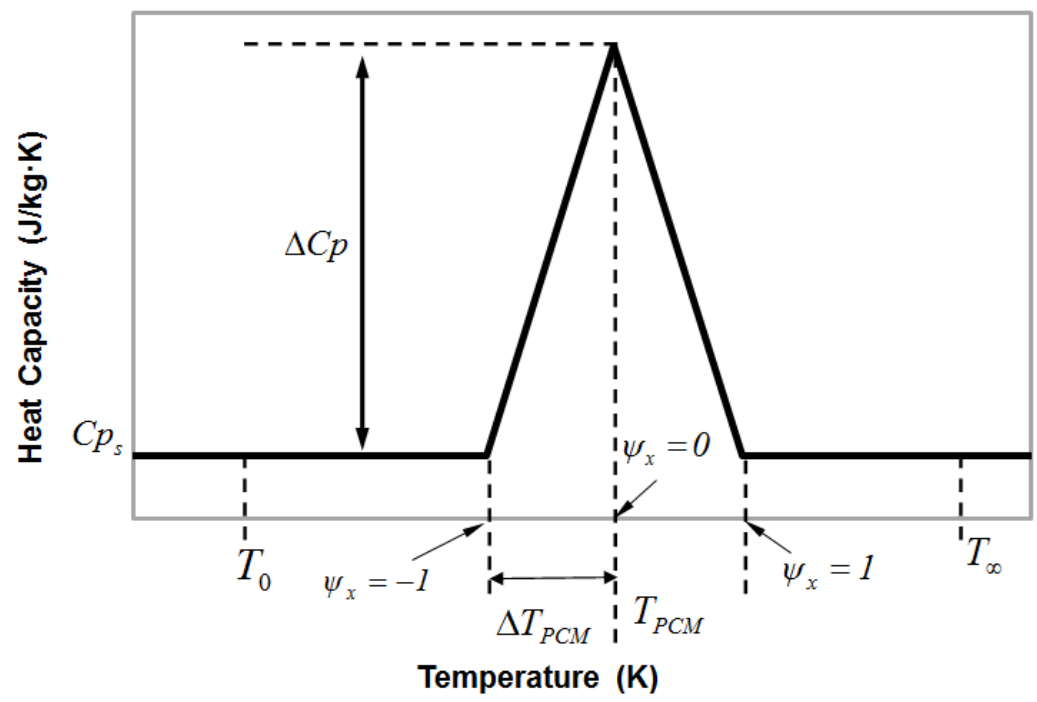

Figure 3: Temperature dependency of the effective specific heat of the phase change materia 


\section{Empirical correlation without the effect of phase change}

\subsection{Significant parameters}

Five parameters were selected to build the correlation for a phase stabilized material wall:

$$
N u^{P S M}=N u^{P S M}\left(\operatorname{Re}_{x}, \theta_{x}, A, B, C\right)
$$

Those parameters intend to characterize:

- The flow regime

- The advance in the heating/cooling process

- The thermal and geometrical properties of the plate

The first parameter is the local Reynolds number $R e_{x}$. It is an image of the flow regime. It is covered in the range $\left[1.10^{5} ; 2.10^{6}\right]$ for a transitional and turbulent flow:

$$
\operatorname{Re}_{x}=\frac{u_{\infty} \cdot x}{v}
$$

The second parameter $\theta_{x}$ represents the progression of the plate heating/cooling process, at the height $x$. Since there are temperature gradients across the plate thickness, it is based on the temperature at the middle of the half plate $T_{m}$ (Figure 1c). The definition of $\theta_{x}$ is given in Eq. 7. It is equal to zero all over the plate at the beginning of the simulation and it is equal to one all over the plate at the end of the simulation.

$$
\theta_{x}=\frac{T_{m}(x)-T_{0}}{T_{\infty}-T_{0}}
$$

Even if $\theta_{x}$ is defined in the range [0;1], the Nusselt correlation was built from simulation results with $\theta_{x} \in[0.05 ; 0.95]$. Indeed, when $\theta_{x}$ approaches one, the convective coefficient is undefined because the plate and the air flow have the same temperature.

The remaining parameters $A, B$ and $C$ take into account the thermal and geometrical characteristics of the plate. $A$ is the volume specific heat ratio between the plate and the air:

$$
A=\frac{\rho_{s} \cdot C p_{s}}{\rho \cdot C p}
$$

$B$ is the ratio between an estimation of the velocity boundary layer thickness $\delta_{x}=5 \sqrt{v x / u_{\infty}}$, and the thickness of the plate $e$ :

$B=\frac{\delta_{x}}{e}$

${ }_{C}$ is the thermal conductivity ratio between the plate and the air: 
$C=\frac{k_{s}}{k}$

Maximum and minimum values of the different physical quantities are shown on Table 1. They were chosen according to Cabeza et al. [23]. Using the parameters from Table 1 , the valid ranges of $R e_{x}, \theta_{x}, A, B$, and $C$ are summarized in Table 2.

Table 1. Minimum and maximum values of the used parameters

\begin{tabular}{ccc} 
& Min. value & Max. value \\
\hline$u_{\infty}\left[\mathrm{m} \mathrm{s}^{-1}\right]$ & 2 & 12 \\
$\rho_{s}\left[\mathrm{~kg} \mathrm{~m}^{-3}\right]$ & 750 & 2200 \\
$C p_{s}\left[\mathrm{~J} \mathrm{~kg}^{-1} \mathrm{~K}^{-1}\right]$ & 500 & 4000 \\
$e[\mathrm{~m}]$ & 0.002 & 0.02 \\
$k_{s}\left[\mathrm{~W} \mathrm{~m}{ }^{-1} \mathrm{~K}^{-1}\right]$ & 0.1 & 2.5
\end{tabular}

Table 2. Valid range of the dimensionless groups used

\begin{tabular}{ccc} 
& Min. value & Max. value \\
\hline $\mathrm{Re}_{\mathrm{x}}$ & $1 \cdot 10^{5}$ & $2 \cdot 10^{6}$ \\
$\theta_{x}$ & 0.05 & 0.95 \\
$A$ & 307.8 & 7222 \\
$B$ & 0.125 & 14.05 \\
$C$ & 4.17 & 104.2
\end{tabular}

\subsection{Formulation of the Nusselt number}

The structure of the correlation is presented in Eq. 11. The dimensionless number $\theta_{x}$ is implemented in the form $\left(\theta_{x}+1\right)^{\alpha}$ because at the beginning of the transient process $\left(\theta_{x}=0\right)$, the heat transfer coefficient is neither equal to zero, nor equal to infinity:

$$
N u_{x, t}^{P S M}=\alpha_{0} \cdot \mathrm{Re}_{x}^{\alpha_{1}} \cdot\left(\theta_{x}+1\right)^{\alpha_{2}} \cdot A^{\alpha_{3}} \cdot B^{\alpha_{4}} \cdot C^{\alpha_{5}}
$$

The software Design-Expert V8 for design of experiment (DOE) defined a set of 31 different simulations to be carried out for the determination of the five constants $\alpha_{i}$. The results of those simulation were then introduced in the R 2.14 statistical software, which calculated the $\alpha_{i}$ using a Least Squares Method.

\subsection{Results}

The correlation obtained after using the Least Squares Method is given in Eq. 12. The average deviation between the simulated Nusselt numbers and the Nusselt values calculated from the correlation is $2.4 \%$. 
$N u_{x, t}^{P S M}=0.036 \cdot \frac{\operatorname{Re}_{x}^{0.78} \cdot\left(\theta_{x}+1\right)^{0.18}}{A^{0.002} \cdot B^{0.01} \cdot C^{0.002}}$

It can be seen in the previous equation that the variation of the parameters $A, B$ and $C$ does not affect significantly the Nusselt number. The authors propose to use an empirical correlation which depends only on $R e_{x}$ and $\theta_{x}$. This new correlation presents an average deviation of $2.5 \%$ from the numerical results.

$N u{ }_{x, t}^{P S M}=0.037 \cdot \operatorname{Re}{ }_{x}^{0.78} \cdot\left(\theta_{x}+1\right)^{0.18}$

\section{Nusselt perturbation due to the phase change}

\subsection{Significant parameters}

A set of 5 dimensionless numbers were defined to describe the phase change perturbation $f^{P C M}$ on the Nusselt evolution:

$$
f^{P C M}=\frac{N u_{x, t}^{P C M}}{N u^{P S M}}=f^{P C M}\left(R e_{x}, S t, S_{C p}, P, \psi_{P C M}\right)
$$

Those parameters intend to characterize:

- The flow regime

- The thermal behavior of the PCM during the phase change

- The advance in the melting/solidification process

The three parameters $S t, S_{c p}$ and $P$ describe the properties of the plate material during the phase change. They characterize the temperature dependency of the PCM effective heat capacity (Cp curve) shown in Figure 3.

$S t$ is the Stefan number based on the temperature difference $T_{\infty}-T_{0}$. The effect of this parameter on the Cp curve is shown in Figure 4a.

$$
S t=\frac{C p_{s}\left(T_{\infty}-T_{0}\right)}{\Delta H_{\text {fusion }}}
$$

The parameter $S_{C p}$ is defined in Eq.16 as the ratio between the increase of specific heat at the phase change temperature and the specific heat in the solid or liquid region (see Figure 3). Figure $4 \mathrm{~b}$ shows the variation of the Cp curve when $S_{C p}$ varies (constant $S t$ ).

$$
S_{C p}=\frac{\Delta C p}{C p}
$$

$P$ fixes the position of the phase change temperature $T_{P C M}$ in the overall process of heating or cooling $\left[T_{0} ; T_{\infty}\right]$. It is defined in Eq. 17. Figure 4c shows the effect of $P$ on Cp curves.

$$
P=\frac{T_{P C M}-T_{0}}{T_{\infty}-T_{0}}
$$




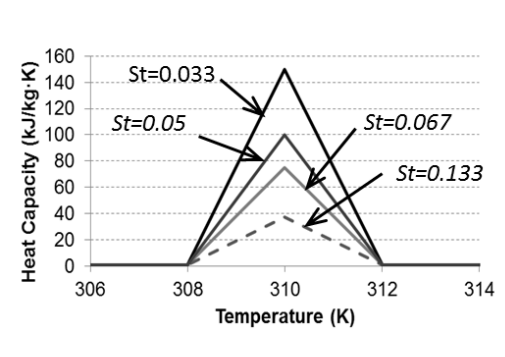

(a)

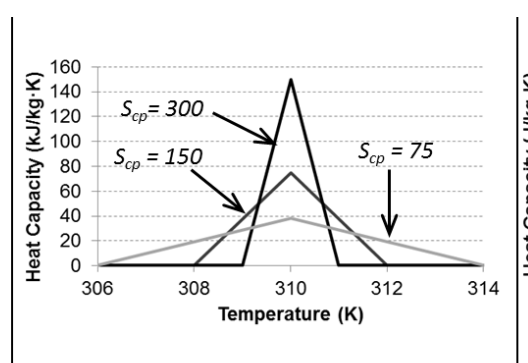

(b)

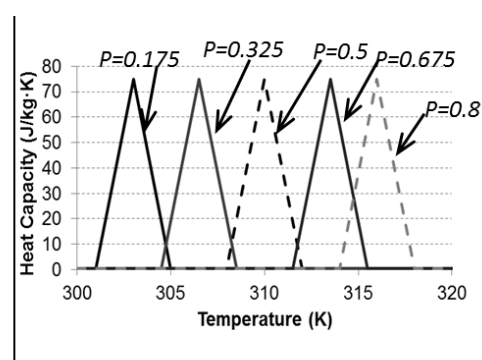

(c)

Figure 4: Variation of the specific heat curve with (a) St, (b) SCp and (c) P

The last dimensionless number $\psi_{x}$ characterizes the advance in the melting/solidification process at the height $x$. Similarly to the parameter $\theta_{x}$, it is based on the temperature at the middle of the half plate $T_{m}$. The expression of $\psi_{x}$ is given by Eq. 18. It has to be multiplied by -1 when used in a fusion process.

$\psi_{x}=\frac{T_{m}(x)-T_{P C M}}{\Delta T_{P C M}}$

Notice that $\psi_{x}$ is equal to zero when the specific heat of the plate is maximum, at $T_{m}(x)=T_{P C M}$. It is equal to -1 at the beginning of the fusion/solidification process and it is equal to 1 at the end of the phase change (Figure 3). The bounds of $\psi_{x}$ depend on the initial and final temperatures $T_{0}$ and $T_{\infty}$.

The studied ranges of the previous parameters are shown in Table 3.

Table 3. Valid range of the parameters used in the correlation for $f^{P C M}$

\begin{tabular}{ccc} 
& Min. value & Max. value \\
\hline$S t$ & 0.033 & 0.14 \\
$P$ & 0.175 & 0.8 \\
$S_{C p}$ & 72 & 300
\end{tabular}

\subsection{Simulation Results}

Table 4 summarizes the set of numerical simulations which have been performed to determine $f^{P C M}$. The numerical results were analyzed through the evolutions of $f^{P C M}$ over $\psi_{x}$.

The curves $f^{P C M}\left(\psi_{x}\right)$ for simulations $\mathrm{C} 1$ to $\mathrm{C} 14$ are shown in Figure 5 . For $\psi_{x} \leq-1$, the phase change has not started yet, hence there is no perturbation on the Nusselt number and $f^{P C M}=1$. During the increasing part of the specific heat curves $\left(-1 \leq \psi_{x} \leq 0\right)$, the phase change has no effect on the Nusselt number, and the perturbation remains equal to 1 . The value of $f^{P C M}$ starts to deviate when the plate reaches the temperature $T_{P C M}$. First, it can be observed an increase of $f^{P C M}$ when $\psi_{x}>0$ until $\psi_{x}=\psi_{c r}$. Finally, $f^{P C M}$ stabilizes at a maximum value $f_{\infty}$. 
Table 4. Set of numerical simulations carried out to define the phase change

\begin{tabular}{ccccc} 
Case & $\mathrm{Re}$ & $\mathrm{St}$ & $\mathrm{S}$ & $\mathrm{P}$ \\
\hline C 1 & $10^{6}$ & 0.034 & 291.8 & 0.5 \\
C 2 & $10^{6}$ & 0.07 & 145.4 & 0.5 \\
C 3 & $10^{6}$ & 0.07 & 145.4 & 0.175 \\
C 4 & $10^{6}$ & 0.07 & 291.8 & 0.5 \\
C 5 & $10^{6}$ & 0.07 & 72.9 & 0.5 \\
C 6 & $10^{6}$ & 0.07 & 205.3 & 0.5 \\
C 7 & $10^{6}$ & 0.14 & 72.2 & 0.5 \\
C 8 & $10^{6}$ & 0.51 & 194.2 & 0.5 \\
C 9 & $4.1 \cdot 10^{5}$ & 0.07 & 145.4 & 0.325 \\
C 10 & $8.2 \cdot 10^{5}$ & 0.07 & 145.4 & 0.325 \\
C 11 & $10^{6}$ & 0.07 & 145.4 & 0.325 \\
C 12 & $1.6 \cdot 10^{6}$ & 0.07 & 145.4 & 0.325 \\
C 13 & $10^{6}$ & 0.07 & 145.4 & 0.675 \\
C 14 & $10^{6}$ & 0.07 & 145.4 & 0.8
\end{tabular}

From Figure 5a, it can be seen that the maximum perturbation $f_{\infty}$ decreases when the Stefan number St increases. Thus, the higher is the latent heat, the higher is the phase change perturbation on the convection coefficient.

Figure $5 b$ shows that the maximum perturbation increases with $P$. The perturbation on the convective heat transfer coefficient will be higher if the phase change occurs at the beginning of the heating/cooling process.

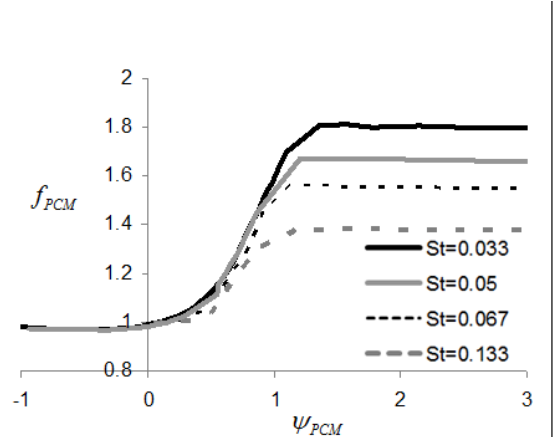

(a)

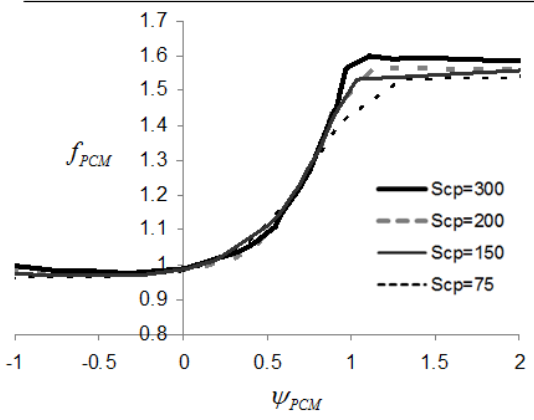

(c)

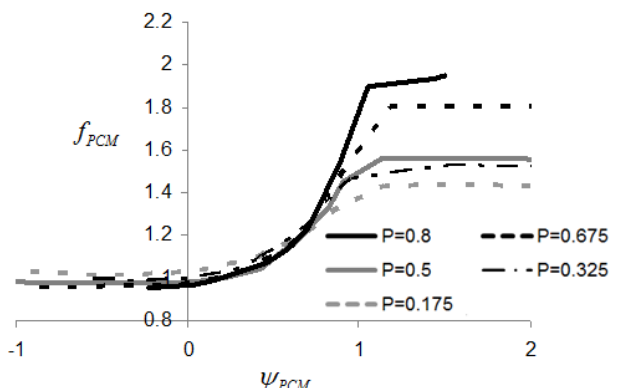

(b)

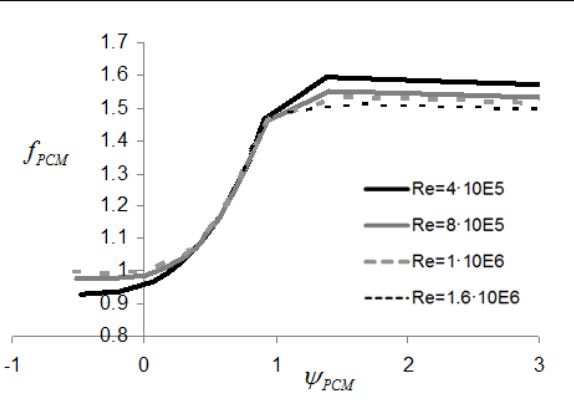

(d)

Figure 5: Effect on the perturbation in the Nusselt evolution due to variation of (a) St, (b) $P$, (c) SCp and (d) Re 
Finally, from Figures $5 \mathrm{c}$ and $5 \mathrm{~d}$, it can be observed that the flow regime (through the Reynolds number) and the width of the phase change temperature range (through the parameter $S_{C_{p}}$ ) have only slight effects on the perturbation curves.

Moreover, Figure 6 compares the perturbation evolutions for a melting and a solidification process. The curves are identical, therefore the same dimensionless model can be used to describe a heating or a cooling process.

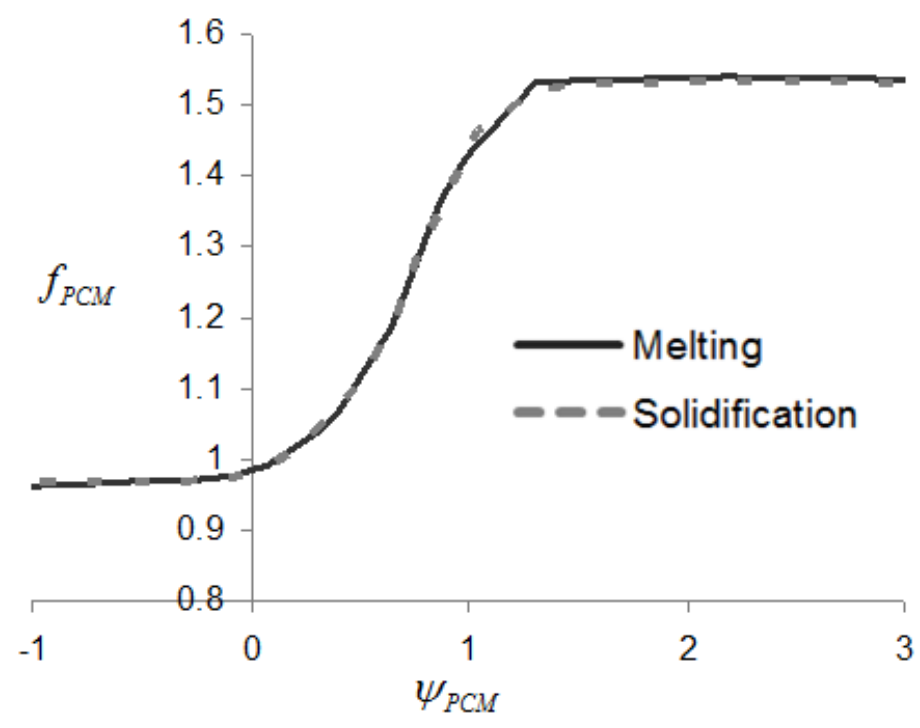

Figure 6: Comparison of the perturbation of the Nusselt number due to solidification and melting process (simulation C2)

\subsection{Formulation of the phase change perturbation}

Given the description of the perturbation curves provided in the last section, the function $f^{P C M}\left(\psi_{x}\right)$ can be expressed as a piecewise function given by Eq. 19 (Figure 7).

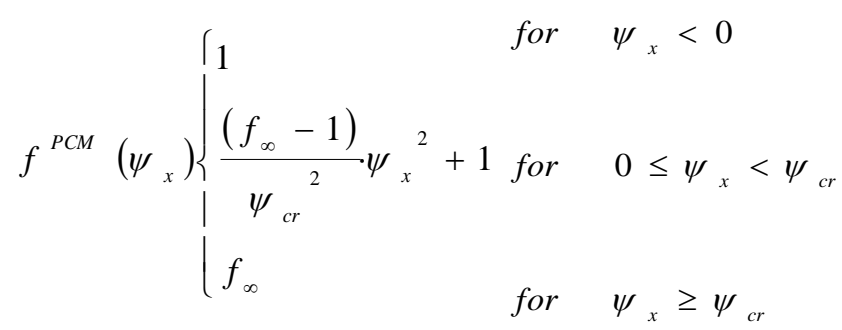

The values of $f_{\infty}$ and $\psi_{c r}$ depend on the parameters $R e_{x}, S t, S_{c p}$ and $P$. They are defined from the simulation results by using a Least Squares Method. 


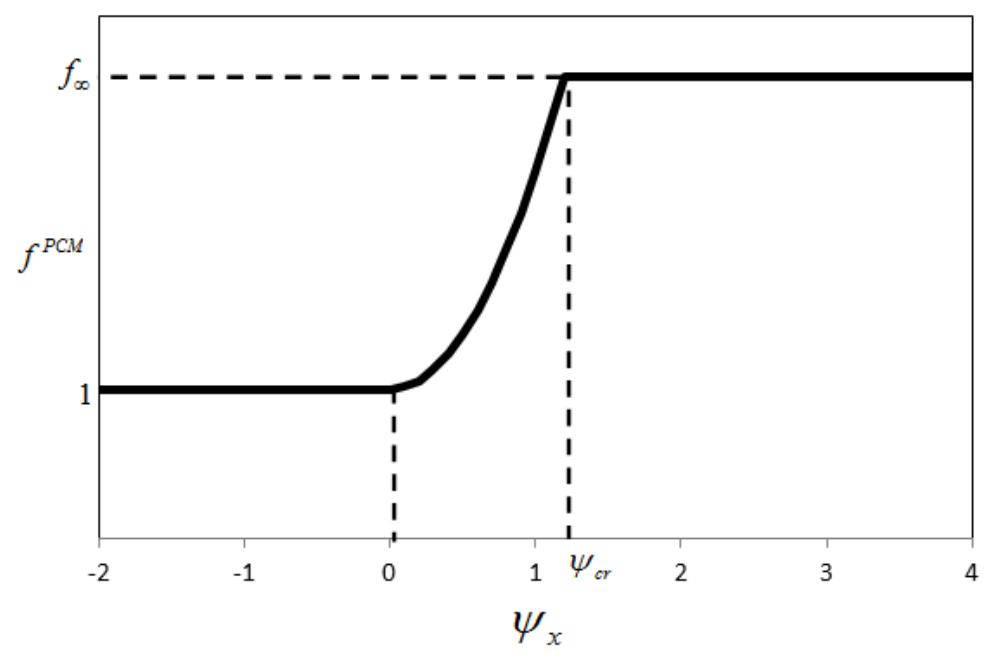

Figure 7: Shape of the perturbation in the Nusselt evolution due to the phase change

The correlation in Eq. 20 calculates the value of the maximum perturbation $f_{\infty}$ with an average error of $2.51 \%$ compared to the CFD results. It can be seen that Reynolds number and $S_{C_{p}}$ are not significant in the correlation. The authors propose a new correlation (Eq. 21) without these two dimensionless numbers. This new correlation presents an average error of $2.84 \%$ with the numerical results.

$$
\begin{aligned}
& f_{\infty}=1.69 \cdot \frac{P^{0.15}}{S t^{0.22} \cdot R e^{0.026} \cdot S_{C p}{ }^{0.033}} \\
& f_{\infty}=1.08 \cdot \frac{\mathrm{P}^{0.14}}{S t^{0.19}}
\end{aligned}
$$

Furthermore, a correlation for $\psi_{c r}$ is provided by Eq. 22. Again, the Reynolds number and the parameter $S_{c_{p}}$ do not influence significantly the values of $\psi_{c r}$ and the correlation presented in Eq. 23, which is based only on $S t$ and $P$, is recommended. The use of Eq. 23 instead of Eq. 22 only increases the average error from $2.35 \%$ to $2.71 \%$.

$$
\begin{aligned}
& \psi_{c r}=0.65 \cdot \frac{\mathrm{P}^{0.10} \cdot \mathrm{R} e^{0.0082} \cdot S_{C p}^{0.039}}{S t^{0.095}} \\
& \psi_{c r}=0.8 \cdot \frac{\mathrm{P}^{0.10}}{S t^{0.13}}
\end{aligned}
$$




\subsection{Discussion}

The particular shape of the curves $f^{P C M}\left(\psi_{x}\right)$ can be understood by analyzing the temperature repartitions in the plate during the cooling and heating processes. For this purpose, Figure 8 contains schematic representations of the plate temperature repartition at the beginning and at the middle of a heating process. The two sketches on the left are for a PSM plate while the two sketches on the right stands for a PCM plate. The velocity boundary layer and the repartition of the heat transfer coefficient are represented on each schema.

The initial conditions are identical for both types of plate: the PCM is solid at the temperature $T_{0}$. Since the air flow is due to external forces, it is not affected by the thermal response of the plate. Hence, the velocity repartition in the fluid is identical for both types of plates and it does not evolve during the heating process.

Nusselt variations are due to evolutions of the thermal boundary conditions at the surface of the plates. For the PSM plate, this evolution is gradual and homogeneous all along the plate. The heat transfer coefficient increases gradually all along the plate until the final temperature is reached.

On the other hand, for the PCM plate, we observe the formation of 5 distinct zones. From the top of the plate (last scheme on Figure 8):

- First zone: the phase change has not started yet and the PCM is still in the solid phase.

- Second zone: this is the first part of the phase change, when the specific heat $C p$ increases with the temperature.

- Third zone: this is the second part of the phase change, when the specific heat $C p$ decreases with the temperature.

- Fourth zone: the phase change is finished. The PCM is in the liquid state, but it has not reached the thermal equilibrium at $T_{\infty}$.

- Fifth zone: the heating process is finished. The PCM is in the liquid state at the temperature $T_{\infty}$.
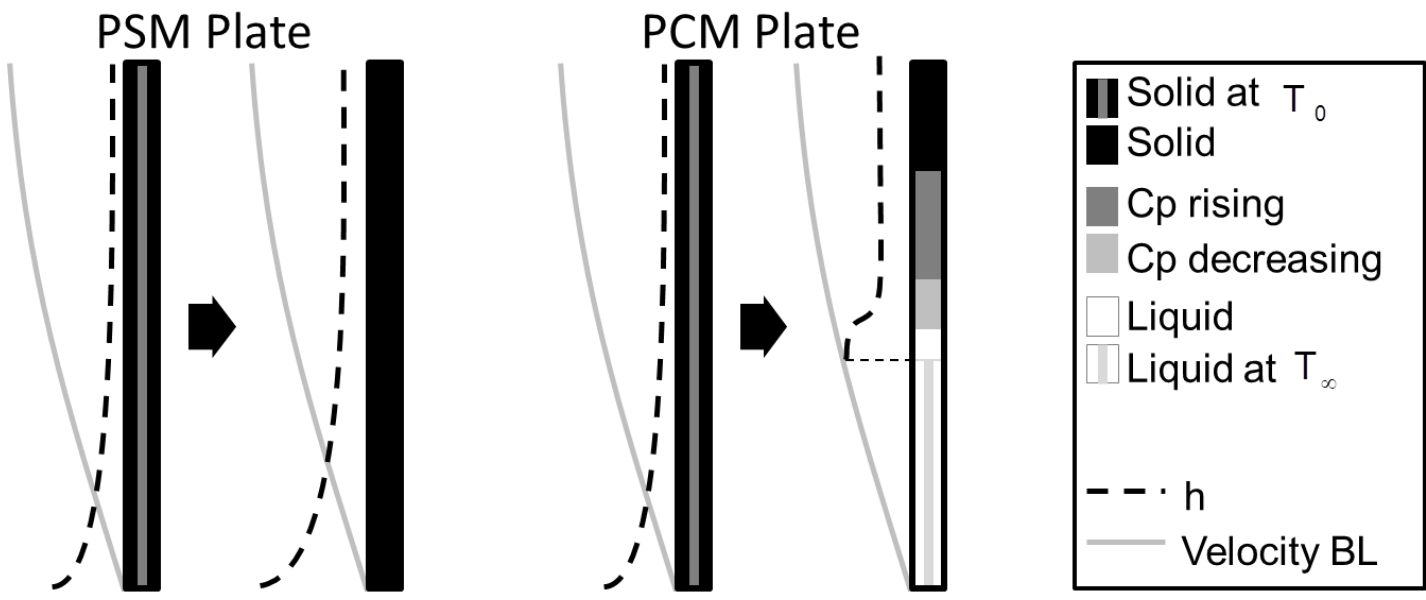

Figure 8: The evolution of the temperature repartition in the plate and its effect on the convection coefficient. On the left: phase stabilized plate. On the right, phase change material plate. 
Thus, the first major difference between PCM and PSM plates is the apparition of the fifth zone near the leading edge. At this location, the plate is in thermal equilibrium with the air flow, hence there is no heat flux at the surface, and the convection heat transfer value has no effect on the system behavior.

The thermal boundary layer starts at the fourth zone. The starting point of the thermal boundary layer induces high values of the heat transfer coefficient. Therefore, the perturbation value is higher in this zone. Since the liquid phase receives heat from the air flow at a high rate, the temperature progression between the end of phase change and the final temperature is fast. Hence, this zone is relatively narrow compared to the other ones.

The phase change occurs in second and third zones. The same amount of heat must be stored in the plate during the first ( $\mathrm{Cp}$ rising) and second ( $\mathrm{Cp}$ decreasing) part of the phase change. However, since the heat transfer rate is higher during the second part, the third zone is narrower.

During the simulation, the sequence of the different zones is shifted toward the upper part of the plate. The last zone which corresponds to the thermal equilibrium grows until it covers the whole wall.

\section{Assessment of the present correlations}

The purpose of the correlations developed in this study is to use them in simplified models of latent heat storage systems. These models include finite volume models for the plates, which are connected to the air flows through convective heat transfer coefficients.

In this section, a nodal model of a half channel is developed. Simulations are performed with different correlations for the convective heat transfer coefficient. The simulation results are compared to CFD results in order to evaluate the improvement due to the use of the correlation built in this study.

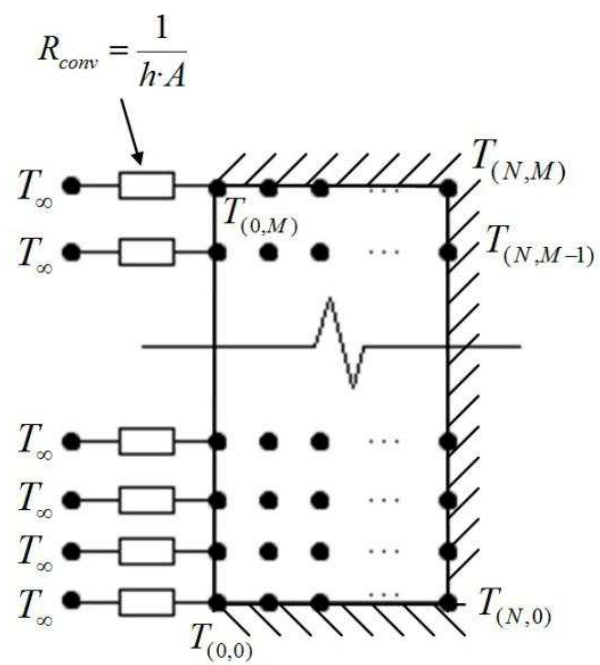

Figure 9 Sketch of the nodal model 


\subsection{Finite volume model}

The energy equation is solved using a fully implicit finite volume method [24]. The transient two-dimensional heat transfer in a PCM plate (Eq. 24) is completed by initial and boundary conditions (Eq. 26-30). L is the height of the plate, $\mathrm{e} / 2$ is the half width of the plate. A sketch of the nodal model is presented in Figure 9.

$$
\begin{aligned}
& \frac{\partial H_{P C M}}{\partial t}=k_{s}\left(\frac{\partial^{2} T}{\partial x^{2}}+\frac{\partial^{2} T}{\partial y^{2}}\right) \quad \text { with } \partial H_{P C M}=\rho_{s} C p(T) \partial T \\
& \text { (i) } \quad t=0 \quad T_{0} \\
& \text { (ii) } \quad x=0 \quad \frac{\partial T}{\partial x}=0 \\
& \text { (iii) } \quad x=L \quad \frac{\partial T}{\partial x}=0 \\
& \text { (iv) } y=0 \quad-k_{s} \frac{\partial T}{\partial x}=h \cdot\left(T_{\infty}-T_{\text {surf }, x}\right) \\
& \text { (v) } y=e / 2 \quad \frac{\partial T}{\partial x}=0
\end{aligned}
$$

A mesh independence study was carried out for the control volume numerical model. A mesh of 400 nodes is used with 40 and 10 nodes in the $x$ and $y$ direction. The system of algebraic linear equations extracted from the finite volume method was solved using the Gauss Seidel iterative algorithm with a time step of $1 \mathrm{~s}$.

The geometric and thermo physical properties of the plate simulated with the nodal model are gathered in Table 5. The PCM plate is heated from $300 \mathrm{~K}$ to $320 \mathrm{~K}$. The melting process starts at $308 \mathrm{~K}$ and finishes at $312 \mathrm{~K}$. The temperature $T_{P C M}$ is $310 \mathrm{~K}$.

\subsection{Simulation results}

One of the main interests in simulating PCM exchangers is to predict the time needed to complete the heating or cooling processes of the plates. As it was previously discussed, the transient evolution of the convective heat transfer coefficient is not explicitly expressed through time, but through the dimensionless parameters $\theta_{x}$ and $\psi_{x}$, which represent the advances in the heating/cooling and fusion/solidification process. In this section, all the results are presented as functions of time. 


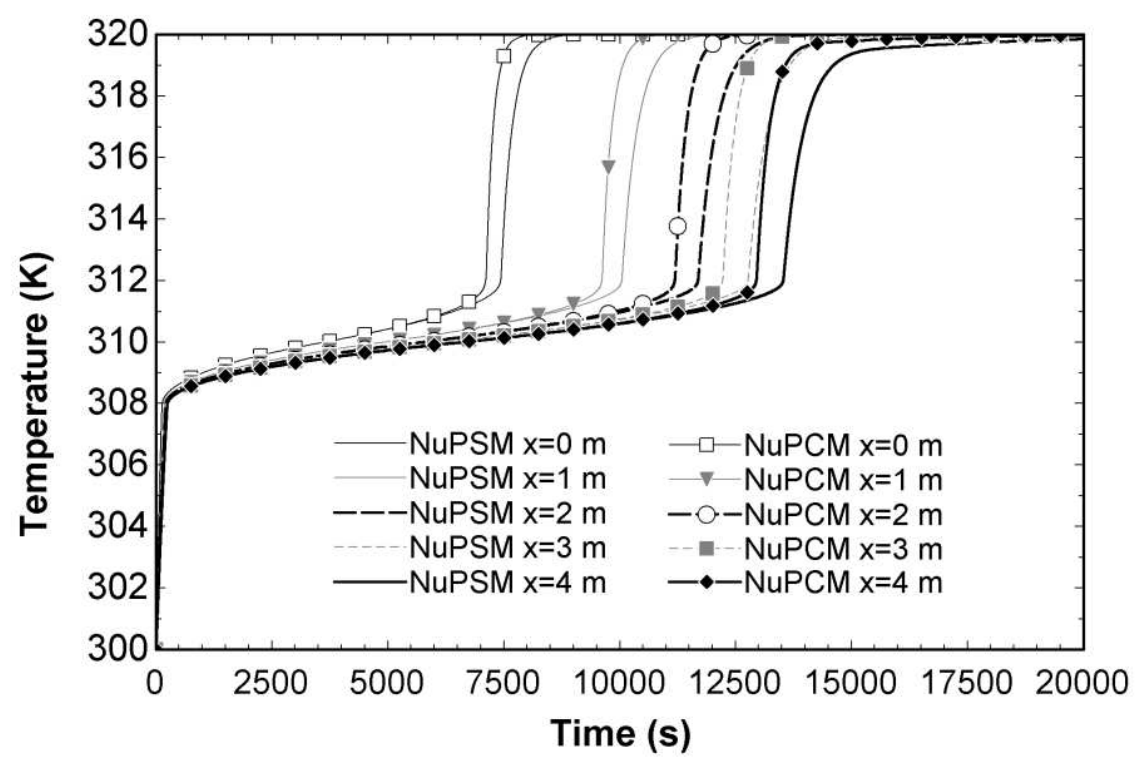

Figure 10: Evolution of the temperature at the middle of the half PCM plate, at different heights. Comparison between the correlations with and without PCM perturbation.

Figure 10 presents the difference in the thermal evolution of the central node at different heights, when the numerical model uses the given base empirical correlation, $\mathrm{Nu}^{P S M}$ (Eq. 13) or the correlation ${ }^{P u}{ }^{P C M}$ which takes into account the perturbation due to the phase change $f^{P C M}$ (Eq. 19, 21, 23).

As it was expected, no differences were found before the phase change temperature peak $(310 \mathrm{~K})$, since the perturbation only acts with positives values of $\psi_{x}$ (Eq. 21). After the peak, the incidence in the thermal evolution of the PCM plate due to perturbation is significant and grows along the height of the plate. The time needed to melt the node at $x=4 \mathrm{~m}$ is reduced around $5 \%$ from the time of the overall process when the perturbation due to phase change is used.

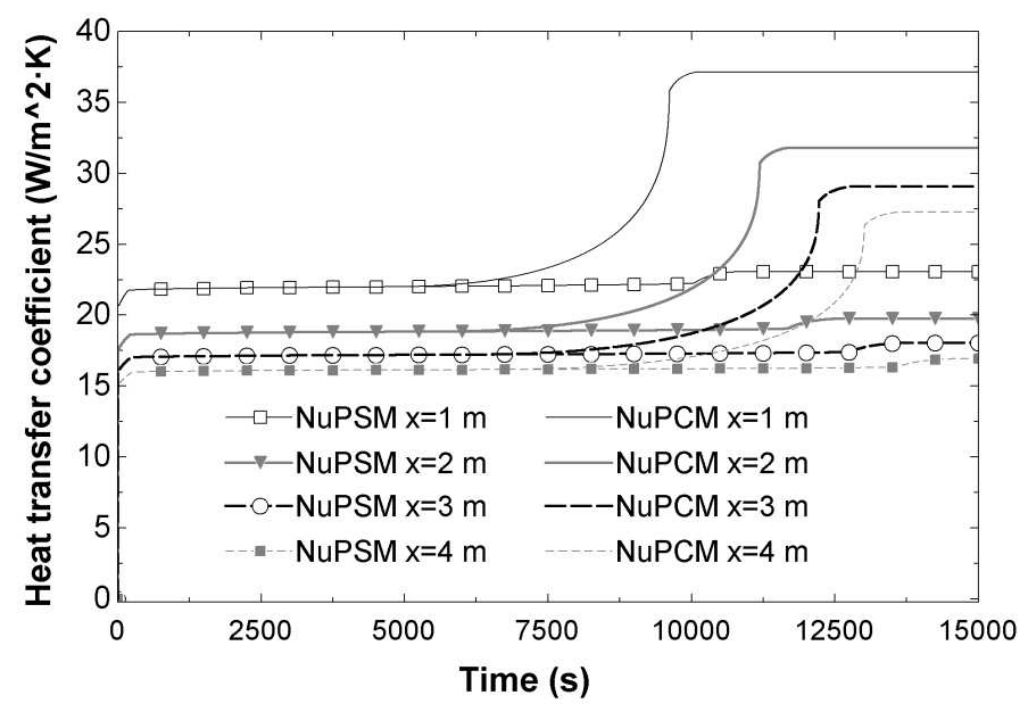

Figure 11 Evolution of the heat transfer coefficient at different heights of the PCM plate. Comparison between the correlations with and without PCM perturbation. 


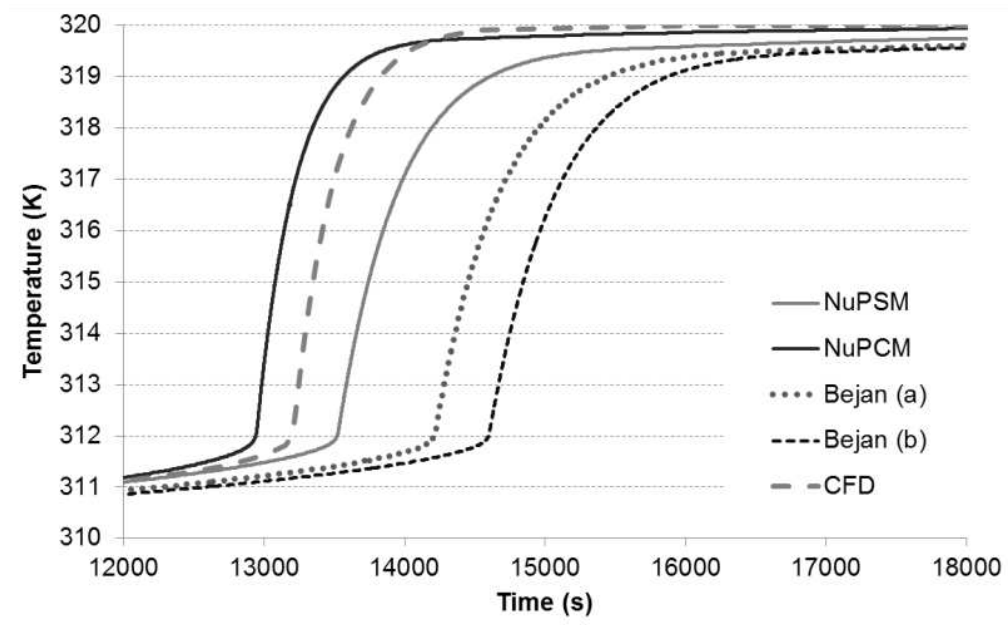

Figure 12 Evolution of the temperature at the top of the PCM plate. Comparison between CFD results and nodal model results with different empirical correlations

Time evolutions of the convective heat transfer coefficient, with and without the perturbation, are presented in Figure 11. As it was previously mentioned, there is no difference between the models before the phase change peak. However, after the phase change peak two distinct behaviors are observed. For the simulations which use the PCM perturbation on $\mathrm{h}$, the heat transfer coefficient grows gradually and reaches a plateau at $\psi_{x}=\psi_{c r}$. For the other simulations, the results show a slight jump of the $h$ coefficient after the phase change is finished. This is due to a fast evolution of the parameter $\theta_{x}$ in the liquid phase.

The thermal load of a PCM wall is finished when the top part of the plate has reached the final temperature $T_{\infty}$. The Figure 12 shows the time evolution of the temperature at the central node at the top of the half plate, for different simulations using different correlations of the heat transfer coefficient. The results are compared to the CDF results. It can be seen that the empirical correlation provided in this work presents better agreement with the CFD results in comparison to the correlation provided by Bejan [20], especially the model which considers the perturbation in the Nusselt number due to the phase change.

Table 6 presents the time needed for each correlation to cover the $95 \%$ of the overall heating process $(319 \mathrm{~K})$ in the analyzed node and compares this time against the time needed in the CFD model (13800 s). It is important to highlight that the model which uses the perturbations not only finish the phase change earlier (3h36min40s from 3h46min40s) but it achieve steady state conditions earlier by increasing the heat transfer coefficient at the studied positions.

\section{Conclusions}

The present study assessed for the first time the issue of forced convective heat transfer along PCM plates. Its major achievements are not only practical, with 
the production of accurate correlations for latent heat storage systems, but also theoretical, with the first analysis of how the temperature repartitions in PCM plates during heat storage processes, affects the convective heat transfer coefficients.

From the practical point of view, the correlations for a PCM plate, and the correlation for the PSM plate are original. These correlations will be suitable for the simulation of sensible and latent heat storage systems, in a wide valid range of the different non dimensional numbers. The use of these correlations in a nodal model will save computational costs compared to the use of computational fluid dynamics simulations.

On the theoretical point of view, the series of simulations which have been performed enabled the authors to have a first understanding of how the plate material phase change affects the convective heat transfer. They clearly showed the presence of a zone, at the bottom of the plate, in which the wall material is in thermal equilibrium with the flow. The presence of this zone delays the starting point of the thermal boundary layer, which leads to heat transfer enhancement at this location.

Obviously, the present study does not pretend to cover the whole subject of forced convection along PCM plates. The correlation will have to be compared to experimental results in the future. And a theoretical analysis of the Navier Stokes equations might be necessary to provide further interpretations of the plate behavior near a flow with time dependent temperature.

\section{Acknowledgments}

This work was supported by the "Corporación Tecnológica de Andalucía" by means of the project "MECLIDE-Soluciones estructurales con materiales especiales para la climatización diferida de edificios" with the colaboration of DETEA. The work was partially funded by the Spanish government (ENE201122722) and the European Union (COST Action COST TU0802). The authors would like to thank the Catalan Government for the quality accreditation given to their research group (2009 SGR 534).

\section{References}

[1] I. Dincer, M.A. Rosen, Thermal energy storage, systems and applications, John Wiley \& Sons, United Kingdom, 2002.

[2] H. Mehling, L.F. Cabeza, Heat and cold storage with PCM. An up to date introduction into basics and applications, Springer-Verlag Berlin Heidelberg, Germany, 2008.

[3] H.Ö. Paksoy, Thermal Energy Storage for Sustainable Energy Consumption. Fundamentals, Case Studies and Design. NATO Science Series, Springer,Turkey, 2007.

[4] F. Kuznik, J. Virgone, K. Johannes, In-situ study of thermal comfort enhancement in a renovated building equipped with phase change material wallboard, Renewable Energy 36 (2011) 1458-1462. 
[5] F. Kuznik, J. Virgone, K. Johannes, Development and validation of a new TRNSYS type for the simulation of external building walls containing PCM, Energy and Buildings 45 (2010) 1004-1009.

[6] A. Castell, I.Martorell, M.Medrano, G.Pérez, L.F. Cabeza, Experimental study of using PCM in brick constructive solutions for passive cooling, Energy and Buildings 42 (2010) 534-540

[7] J. Borderon, S. Clouseau, R.Cantin, J. Virgone, Simulation of a building equipped with a PCM/air heat exchanger, PALENC 2010, 1-10, Rhodes, 2010.

[8] J. Borderon, J. Virgone, R. Cantin, Numerical analysis of ventilation temperatures regulation by energy storage in phase change materials, ISES Solar World Congress, Kassel, 2011.

[9] J. Borderon, J. Virgone, R. Cantin, K. Kuznik, Full-scale study of a building equipped with a multi-layer rack latent heat thermal energy store system, HVAC\&R, Taylor and Francis, Vol. 17, N. 4, (2011) 566 - 576.

[10] D. Faggembauu, M. Costa, M. Soria, A. Oliva, Numerical analysis of the termal behaviour of ventilated glazed facades in Mediterranean climates. Part I: development and validation of a numerical model, Solar Energy 75 (2003) 217228.

[11] D. Faggembauu, M. Costa, M. Soria, A. Oliva, Numerical analysis of the termal behaviour of ventilated glazed facades in Mediterranean climates. Part II: applications and analysis of results, Solar Energy 75 (2003) 229-239.

[12] Liu M, Saman W, Bruno F, Validation of a mathematical model for encapsulated phase change material flat slabs for cooling applications, Applied Thermal Engineering 31 (2011) 2340-2347.

[12] J. Wei, Y. Kawaguchi, S. Hirano, H, Takeuchi, Study on a PCM heat storage system for rapid heat supply, Applied Thermal Engineering 25 (2005) 2903-2920.

[13] P. Dolado, A. Lazaro, J.M. Marin, B. Zalba, Characterization of melting and solidification in a real scale PCM-air heat exchanger: Numerical model and experimental validation, Energy Conversion and Management 52 (2011) 18901907.

[14] V. Gnielinski, New equations for heat and mass transfer in turbulent pipe and channel flow, International Journal of Chemical Engineering 16 (1976) 359368.

[15] G. Hed, R. Bellander, Mathematical modelling of PCM air heat exchanger, Energy and Buildings 38 (2006) 82-89.

[16] J.P. Holman, Heat Transfer, eighth ed., McGraw-Hill Companies, USA, 1998.

[17] D. David, F. Kuznik, J. Roux, Numerical study of the influence of the convective heat transfer on the dynamical behavior of a phase change material wall, Applied thermal Engineering 31 (2011) 3117-3124.

[18] D. David, Étude Experimental study of the natural convection in the vicinity of walls containing phase change materials PhD. Centre de Thermique de Lyon, France, 2010 (In French). 
[19] A. Bejan, Convection heat transfer, second ed., John Wiley \& Sons, USA, 1995.

[20] P. Lamberg, R. Lehtiniemi, A. H, Numerical and experimental investigation of melting and freezing processes in phase change material storage, International Journal of Thermal Sciences 43 (2004) 277-287.

[21] M.M. Farid, A new approach in the calculation of heat transfer with phase change, $9^{\text {th }}$ International Congress on Energy and Environment, 1-19, Miami, 1989.

[22] L.F. Cabeza, A. Castell, C. Barreneche, A. de Gracia, A.I. Fernández, Materials used as PCM in thermal energy storage in buildings: A review, Renewable and Sustainable Energy Reviews 15 (2011) 1675-1695.

[23] S. Patankar, Numerical Heat Transfer and Fluid Flow, Hemisphere Publications, USA, 1980.

\section{Nomenclature}

$B$

Dimensionless number used in the Nusselt correlation Dimensionless number used in the Nusselt correlation

$C$ Dimensionless number used in the Nusselt correlation

$C_{f, x}$ Local skin-friction coefficient, dimensionless

$C p$

Specific heat, $\mathrm{J} \mathrm{kg}^{-1} \mathrm{~K}^{-1}$

$e$

Thickness of the solid plate, $m$

Perturbation in the Nusselt correlation due to phase change, dimensionless

St

Stefan Number

$H_{P C M}$

Enthalpy of the PCM, $\mathrm{kJ} \mathrm{kg}^{-1}$

$h$

Convective heat transfer coefficient, $\mathrm{W} \mathrm{m}^{-2} \mathrm{~K}^{-1}$

$k$

Thermal conductivity, $\mathrm{W} \mathrm{m}^{-1} \mathrm{~K}^{-1}$

$L \quad$ Total height of the plate, $\mathrm{m}$

$\mathrm{Nu}{ }^{P S M}$

Nusselt number of an air flor over a phase stabilized material

$N u \underset{x, t}{P C M}$

Nusselt number of an air flow over a phase change material

$P$

Dimensionless number used in the perturbation correlation

PCM

Phase change material

PSM

Phase stabilized material

$\mathrm{Pr} \quad$ Prandlt number

$\mathrm{Re} \quad$ Reynolds number

$S_{C p}$

Dimensionless number used in the perturbation correlation 


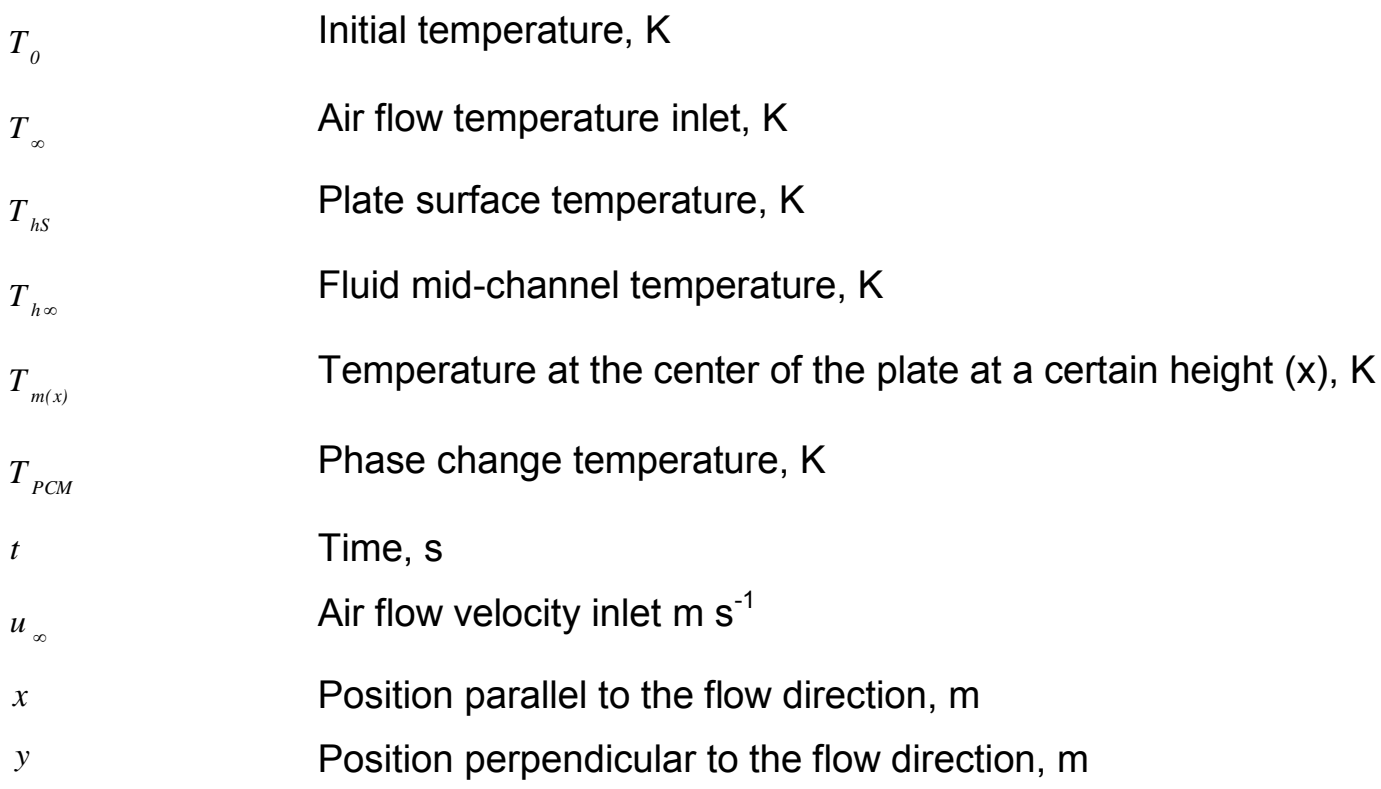

